\title{
Morphological Image Reconstruction with Criterion from Labelled Markers
}

\author{
Damián Vargas-Vazquez, Jose Crespo, and Victor Maojo \\ Facultad de Informática \\ Universidad Politécnica de Madrid \\ 28660 Boadilla del Monte (Madrid), Spain \\ damian@infomed.dia.fi.upm.es
}

\begin{abstract}
In Mathematical Morphology, the reconstruction of images from markers has proven to be useful in morphological filtering and image segmentation. This work investigates the utilization of a criterion in the reconstruction process, whose utilization in the problem of the image reconstruction from an image marker has been partially treated elsewhere. This work further investigates this idea and extends it to the problem of image reconstruction from labelled markers. In the binary case, this allows us to compute the modified influence zones associated to the set of labelled markers. A significant difference with the usual case (i.e., the "normal" influence zones) is that we generally do not obtain a whole partition of the space, because the criterion added to the reconstruction process causes that some points or pixels are not recovered. In addition, in this paper we consider the gray-level case, and we use the reconstruction with criterion to separate regions from a non-binary input image. This input image is considered as a topographic relief (similarly as in a normal watershed); however, the flooding mechanism is modified by the reconstruction criterion. The benefit is that we can control to some extent how the flooding proceeds and, therefore, how image region shapes are recovered.
\end{abstract}

Keywords: Mathematical Morphology, segmentation, flat zones, labelled markers, reconstruction with criterion.

\section{Introduction}

In Mathematical Morphology [10] [11] [14] 6] [9], the use of reconstruction algorithms has been successfully used in the stages of image processing and analysis. Filters by reconstruction [12 [2] 11] 8] 3] [4] [13 have become powerful tools that enable us to eliminate undesirable features without practically affecting desirable ones. These filters are computed by reconstructing a reference image $f$ from a marker image $g$, and they preserve well the shapes of the marked structures.

A new type of transformations - known as transformations with reconstruction criterion - are derived from filters by reconstruction. A modification of the reconstruction process, in particular the inclusion of a criterion, allows us to 
control the shape of some structures while preserving contours and the structures of interest. The main feature of these transformations is that they enable us to obtain intermediate results between the standard morphological opening (or, respectively, closing) and the opening (resp., closing) by reconstruction, and some of their inconveniences can be avoided.

These filters by reconstruction with criterion have been partially treated in [15] [17]. In this paper, we will study the application of the reconstruction criterion to the problem of the reconstruction of an input image from labelled markers (or connected components). In the binary case, we will discuss the main differences that exist with respect to the "non-criterion" case, and we will study how to compute the modified influence zones. As will be discussed, some points are not recovered in the reconstruction process, and the criterion used modifies ultimately the influence zones shapes. In addition, we will apply these ideas to the gray-level case, in which case the reconstruction criterion provides some control about how the "flooding" of the topographical relief constituted by the input image (using terms normally employed in the watershed transformation method) proceeds. As expected from the binary case discussion, not all pixels of the input image will belong to the computed regions, since the criterion causes, in certain situations, that some pixels are not reached by the reconstruction mechanism.

Section 2 discusses some aspects of filters by reconstruction with criterion. Section 3 considers the problem of image reconstruction (with criterion) using labelled markers in both the binary case (Section 3.2) and the gray level case (Section 3.3), where differences with the normal reconstruction (i.e., where no criterion is employed) will be highlighted.

\section{Openings and Closings with Reconstruction Criterion}

The process to build these types of transformations involves the use of a reference image and a marker image. Thus, a reconstruction process of a marker image inside a reference image is made (as is the case in transformations by reconstruction), but a reconstruction criterion is taken into account [15] [17].

Let $f$ and $g$ be the reference and marker image respectively. We will consider the next propagation criteria:

$$
f \wedge \gamma_{\lambda} \delta_{(1)}(g) \text { and } f \vee \varphi_{\lambda} \varepsilon_{(1)}(g)
$$

the first for the opening case, and the second for the closing case. Note: $\gamma_{\lambda}$ and $\varphi_{\lambda}$ denote, respectively, an opening and a closing of index $\lambda$ (which defines the structuring element size) and $\delta_{(1)}$ and $\varepsilon_{(1)}$ symbolize the elementary dilation and erosion, respectively (which, for example, employ a $3 \times 3$ square structuring element using 8-connectivity). We will refer in the following expressions only to the opening case (dual expressions will apply also to the closing case).

Let us remember that, in the opening by reconstruction, the operation used is $f \wedge \delta_{(1)}(g)$, which is the geodesic dilation. In our case, the opening $\gamma_{\lambda}$ plays the special role of propagation criterion. We have the following inequality:

$$
g<\gamma_{\lambda} \delta_{(1)}(g)<\delta_{(1)}(g)
$$


since $\gamma_{\lambda}$ is an anti-extensive operator. For $\lambda=0$, we have expression $g<$ $\gamma_{\lambda} \delta_{(1)}(g)=\delta_{(1)}(g)$. That is, the propagation process of marker $g$ is realized in the same way as in the opening by reconstruction. However, if $\lambda \geq 1$, although inequality $\gamma_{\lambda} \delta_{(1)}(g)<\delta_{(1)}(g)$ holds ( since $\gamma_{\lambda}$ is anti-extensive), inequality $g \leq \gamma_{\lambda} \delta_{(1)}(g)$ is not necessarily true.

In the case of the opening by reconstruction, when the marker is given by a morphological opening $g=\gamma_{\mu}(f)$ instead of the erosion function $g=\varepsilon_{\mu}(f)$, we can obtain the same result (assuming the structuring element contains the center).

Specifically, when the marker image is given by $g=\gamma_{\mu}(f)$, for $\lambda \leq \mu+1$, the output images of successive iterations of the operation $\gamma_{\lambda} \delta_{(1)}$ are similar to those generated by the successive iterations of $\delta_{(1)}$. However, the reconstruction process changes when the reference image $f$ is used. It is possible to appreciate the propagation criterion given by $\gamma_{\lambda}$ if we iterate the operator $\sigma_{\lambda, f}^{(1)}\left(\gamma_{\mu}(f)\right)=$ $f \wedge \gamma_{\lambda} \delta_{(1)}\left(\gamma_{\mu}(f)\right)$ until idempotence to obtain the opening with reconstruction criterion $\widehat{\gamma}_{\lambda, \mu}$ (and, in a similar way, for operator $\widehat{\varphi}_{\lambda, \mu}$ ):

$$
\widehat{\gamma}_{\lambda, \mu}(f)=\lim _{n \rightarrow \infty} \sigma_{\lambda, f}^{(n)}\left(\gamma_{\mu}(f)\right)=\underbrace{\sigma_{\lambda, f}^{(1)} \sigma_{\lambda, f}^{(1)} \cdots \sigma_{\lambda, f}^{(1)}\left(\gamma_{\mu}(f)\right)}_{\text {until idempotence }} .
$$

In this case, the reference image modifies the reconstruction process of successive iterations of $\gamma_{\lambda} \delta_{(1)}$, where the opening $\gamma_{\lambda}$ restricts the reconstruction to some regions of the reference image $f$.

There are inclusion relationships between the flat zones obtained at the output of the opening (and, respectively, closing) with reconstruction criteria and those at the output of classical opening (respectively, closing) by reconstruction. I.e., each flat zone of the output of an opening with reconstruction criterion is included in a flat zone of the output of the corresponding opening by reconstruction. In fact, there are inclusion relationships between those filters and morphological openings and closings (without reconstruction).

Thus, using a non-connected opening as a marker we can establish a flat zone inclusion relationship. One extreme would be the case of the non-connected opening (used as the marker), and the other extreme would be the case of the classical opening by reconstruction. Between those cases we would find the gradation constituted by the family of the opening with reconstruction criteria, whose criterion allows us to control the reconstruction of flat zones and the resulting inclusion relationships.

Figure 1 and 2 illustrate this. The example in Figure 1 shows the gradation that can be obtained with the opening with reconstruction criteria, whose outputs appear as intermediate results between those of the non-connected filters and of the filters by reconstruction. In the binary case (Figure 2), we can see how we can control the flat zone extension and, if we desire it, separate certain regions in some cases. 


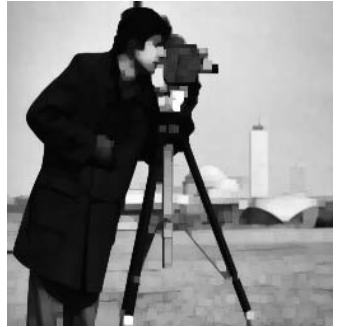

(a)

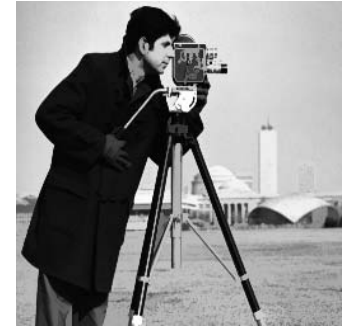

(b)

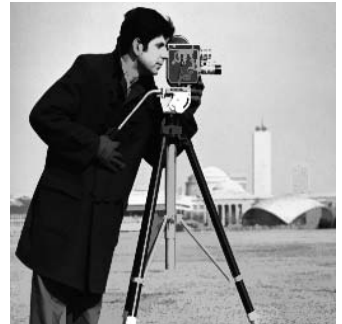

(c)

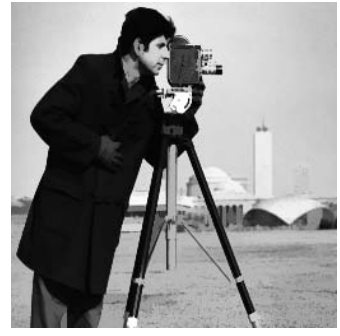

(d)

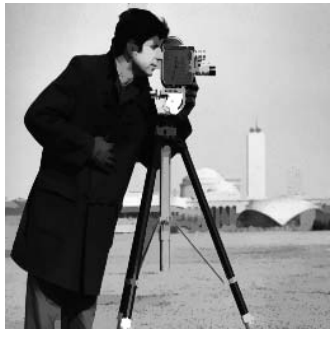

(e)

Fig. 1. (a) Morphological opening $\gamma_{\mu}$ with $\mu=2$; (b) opening by reconstruction $\widetilde{\gamma}_{\mu}$ with $\mu=2$; (c) opening with reconstruction criteria $\widehat{\gamma}_{\lambda, \mu}$ using $\mu=2, \lambda=1$; (d) $\widehat{\gamma}_{\lambda, \mu}$ using $\mu=2, \lambda=2$; and (e) $\widehat{\gamma}_{\lambda, \mu}$ using $\mu=2, \lambda=3$.

\section{Reconstruction with Criterion from Labelled Markers}

In this section we will discuss the reconstruction with criterion from labelled markers. First, we will consider the binary case where we will discuss the computation of influence zones associated with the markers. Afterwards we will apply these concepts to the gray-level image case.

\subsection{General Definitions}

We will consider only digital images in the following. A gray-level image can be represented by a function $f: D \rightarrow L$, where $D$ is a subset of $Z^{2}$ and $L$ is a subset of $Z$ ( $Z$ denotes the set of integers).

A section of $f$ at level $i$ is a set $X_{i}(f)$ defined as: $X_{i}(f)=\{x \in D: f(x) \geq i\}$. In the same way, we may define the set $Z_{i}(f)$ as: $Z_{i}(f)=\{x \in D: f(x) \leq i\}$. We have clearly $X_{i}(f)=\complement\left(Z_{i+1}(f)\right)$, where $\complement$ denotes the complementation operator.

Let $M$ be a set of $D$. For every point $y$ of $M$, we will denote the distance function of $y$ to complementary set $\complement(M)$ as:

$$
\forall y \in M, \quad d(y)=\operatorname{dist}(y, \complement(M))
$$

where $\operatorname{dist}(y, \complement(M))$ is the shortest distance between $y$ and a point of $\complement(M)$.

Let $X \subset D$ be a set, and $x, y$ two points of $X$. We define the geodesic distance $d_{X}(x, y)$ between $x$ and $y$ as the length of the shortest path (if any) included in $X$ and linking $x$ and $y$. 


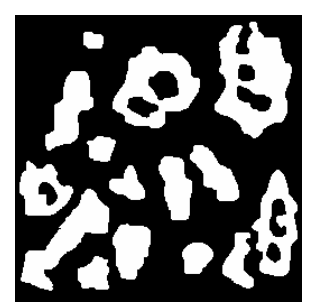

(a)

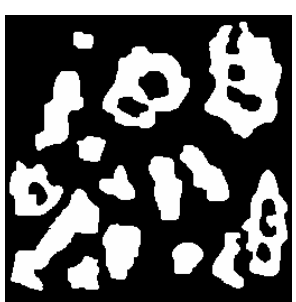

(b)

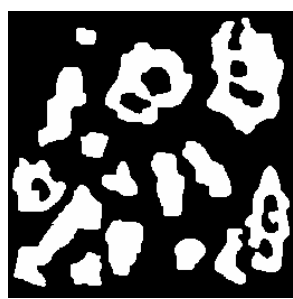

(c)

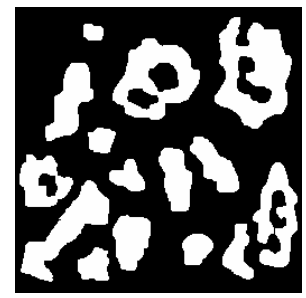

(d)

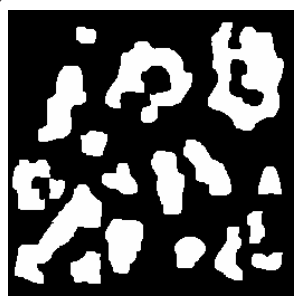

(e)

Fig. 2. (a) Original Image; (b) opening with reconstruction criteria $\widehat{\gamma}_{\lambda, \mu}$ using $\mu=5$, $\lambda=2$; (c) $\widehat{\gamma}_{\lambda, \mu}$ using $\mu=5, \lambda=3$; (d) $\widehat{\gamma}_{\lambda, \mu}$ using $\mu=5, \lambda=4$; and (e) $\widehat{\gamma}_{\lambda, \mu}$ using $\mu=5$, $\lambda=5$.

Suppose now that $M$ is composed of $n$ connected components (markers) $M_{i}$. The geodesic zone of influence $z_{X}\left(M_{i}\right)$ of marker $M_{i}$ is the set of points of $X$ located at a finite geodesic distance from $M_{i}$ that are closer to $M_{i}$ than to any other marker $M_{j}$ :

$$
z_{X}\left(M_{i}\right)=\left\{x \in X: d_{X}\left(x, M_{i}\right) \text { finite, } \forall j \neq i, d_{X}\left(x, M_{i}\right)<d_{X}\left(x, M_{j}\right)\right\} .
$$

The boundaries between the various zones of influence constitute the geodesic skeleton by zones of influence (SKIZ) of $M$ in $X$. We can write:

$$
\mathrm{IZ}_{X}(M)=\bigcup_{i} z_{X}\left(M_{i}\right)
$$

and:

$$
\operatorname{SKIZ}_{X}(M)=X / \operatorname{IZ}_{X}(M) .
$$

where / stands for the set subtraction.

\subsection{Binary Case: Geodesic Distance Modification}

Let $C_{X}(x, y)$ denote the set of paths that link $x$ and $y$. Such a set can be the empty set, in particular if $x$ and $y$ belong to connected disjoint components of $X$. We can write the geodesic distance as:

$$
\begin{gathered}
d_{X}(x, y)=\wedge\left\{\ell(f), f \in C_{X}(x, y)\right\} . \\
d_{X}(x, y)=\infty \quad \text { if } \quad C_{X}(x, y)=\emptyset .
\end{gathered}
$$

where $\ell$ is the length of path of points (number of points). 
Suppose now that we apply an opening with reconstruction criterion $\widehat{\gamma}_{\lambda, g}$ (expression $(3))$ to image $X$. We will modify the geodesic distance $d_{X}(x, y)$ expressions indicated above, and we will use instead $D_{X}(x, y)$, which is defined next:

$$
\begin{aligned}
& D_{X}(x, y)=\wedge\left\{\ell(f), f \in C_{\widehat{\gamma}_{\lambda, g}(X)}(x, y)\right\} . \\
& D_{X}(x, y)=\infty \text { if } C_{\widehat{\gamma}_{\lambda, g}(X)}(x, y)=\emptyset .
\end{aligned}
$$

Note that, instead of paths included in $X$, we are considering paths included in the filter output $\widehat{\gamma}_{\lambda, g}(X)$.

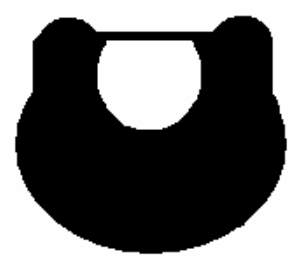

(a)

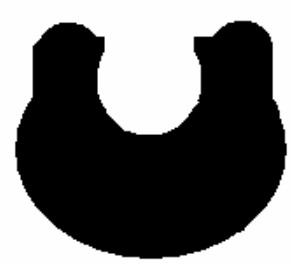

(c)

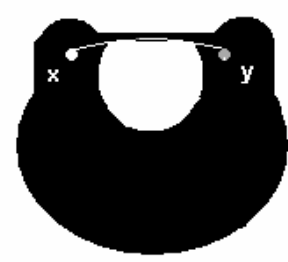

(b)

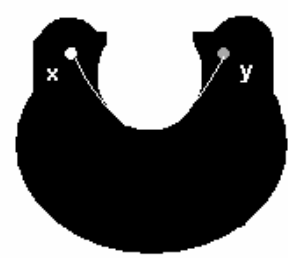

(d)

Fig. 3. Shortest path differences.

Figure 3 illustrates the $D_{X}(x, y)$ concept. Figure 3(a) and 3)(b) display the usual case and the shortest path between a pair of points $x$ and $y$ that belong to $X$. Figure [3(c) visualizes the filter output $\widehat{\gamma}_{\lambda, g}(X)$, and Figure 3(d) displays the shortest path between $x$ and $y$ that is included in $\widehat{\gamma}_{\lambda, g}(X)$. Note that, in this case, $d_{X}(x, y)$ is quite different from $D_{X}(x, y)$.

Now we will consider the problem of computing the influence zones associated to a set of markers. The following expression will define the new $\widehat{z_{X}}\left(M_{i}\right)$ influence zone of marker $M_{i}$ :

$$
\widehat{z_{X}}\left(M_{i}\right)=\left\{x \in \widehat{\gamma}_{\lambda, g}(X): D_{X}\left(x, M_{i}\right) \text { finite, } \forall j \neq i, D_{X}\left(x, M_{i}\right)<D_{X}\left(x, M_{j}\right)\right\} .
$$

where $g=\cup_{i} M_{i}$.

The following figures illustrate the computation of $\widehat{z_{X}}\left(M_{i}\right)$. Figure 4 (a) displays an input set with two markers $M_{1}$ and $M_{2}$. Figure 4(b) and 4(c) display, respectively, the influence zones of $M_{1}$ and $M_{2}$, considering there is only one 


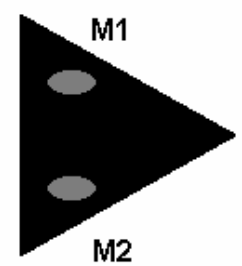

(a)

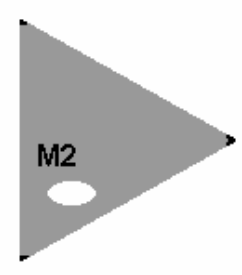

(c)

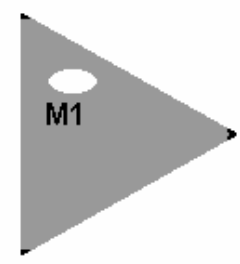

(b)

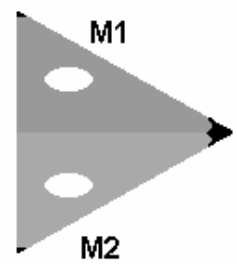

(d)

Fig. 4. Modified influence zones.

marker $\left(M_{1}\right.$ or $\left.M_{2}\right)$. Figure 4(d) displays the influence zones of both markers. Note that there are some (a few) points at the right corner in Figure 4 (d) that do not belong to any influence zones, but that belong to the influence zones in Figure $4(\mathrm{~b})$ or $4(\mathrm{c})$. The reason is that there are some pixels that belong to $\widehat{\gamma}_{\lambda, g}(X)$ when $g=M_{1}$ or $g=M_{2}$, but not when $g=M_{1} \cup M_{2}$.

\subsection{Gray-Level Case}

In this section, we will apply previous concepts to the gray-level case. The input image will be considered as a topographic relief that is flooded (using terms normally used in the watershed method [5] 7] [16] 1]). We will discuss next the expressions of this modified flooding process, which proceeds level by level.

Let the marker image $M_{j}$ be a gray-level image composed of labelled markers $m_{k}$ at time $j$, where $m_{k}$ is a connected component with label $k$, where $k \in$ $\{1, \ldots, N\}$, and $N$ is the number of markers. In $M_{j}$, background pixels (those that do not belong to a labelled marker) has an intensity value of 0 . The initial marker image $M_{0}$ is composed of the set of all the minima of the original image $f$. Then, the next sequence operation compute the boundary image $B$ ( $B$ is a binary image where boundary pixels will have zero intensity value and the rest of them will have the maximum value MAXVALUE of the images under consideration) 1

Initialize counters: $\mathrm{i}=0, \mathrm{j}=1$

Initialize Boundary Image $B: B(x)=$ MAXVALuE $\forall$ pixel $x$

\footnotetext{
${ }^{1}$ For example, for two byte-per-pixel images, this value is equal to 65535 .
} 
For all levels $i$ of $f$ do

$$
T_{i}(f)=\left\{\begin{array}{cl}
\text { MAXVALUE } & \forall x: f(x) \leq i \\
0 & \text { otherwise }
\end{array}\right.
$$

Do

$$
\begin{aligned}
& M_{j}=\left(\gamma_{\lambda} \delta_{(1)}\left(M_{j-1}\right) \wedge T_{i}(f)\right) \vee M_{j-1} / * \text { image marker update } * / \\
& \text { for }(k=1, k \leq N) \text { do } \\
& \text { for all border pixels p of } m_{k} \text { do } \\
& \text { if } \exists p^{\prime} \in N_{G}(p) \text { so that } M_{j}\left(p^{\prime}\right)>0 \text { and } M_{j}\left(p^{\prime}\right) \neq k \\
& \qquad B\left(p^{\prime}\right)=0 / * \mathrm{p} \text { ' is labelled as a boundary pixel } * / \\
& \quad M_{j}=M_{j} \wedge B
\end{aligned}
$$
$\mathrm{i}=\mathrm{i}+1$

where $N_{G}(p)$ is the set of neighbors of a pixel $p$. Note that the inf operation " $M_{j}=M_{j} \wedge B$ " is necessary to prevent the mixing of different markers.

At the end of the process, the boundaries are the separations of the modified catchment basins. Nevertheless, note that some pixels may not be flooded (as discussed in Section 3.2 for the binary case), because of the added reconstruction criteria.

The suggested reconstruction criterion $\gamma_{\lambda} \delta_{(1)}\left(M_{j-1}\right)$, introduced by the transformations described in Section [3, allows us to have some flexibility to separate flat or connected zones, limiting the immersion process to certain zones.

In Figure 5], we show an application of this modified flooding transformation for particle extraction in a medical gray-level images. Figure 5 a) displays an input image, and Figure 5(b) show the markers highlighted in white over the original (note that the background marker is also displayed). If we only want to segment the marked cells as unique regions, the watershed transformation will not be the most suitable option, because the flooding process will recover all overlapping components (including the small particle at the upper-left corner joined by a thin structure that, in this case, is desired to be extracted separately) as is showed in Figure 5 (c).

Figure 5(d) visualizes the image region recovered by the transformation presented above. As we can see, the reconstruction criterion added can prevent the undesired mixing of overlapping particles (as is the case of the particle at the upper-left corner). Note that, in this case, pixels that are not assigned to the particle markers will be ultimately flooded by the background marker.

\subsection{Conclusion}

This paper has investigated the utilization of a criterion in the reconstruction process and has applied it to the problem of image reconstruction from labelled markers. In the binary case, we have studied the differences that exist in the 


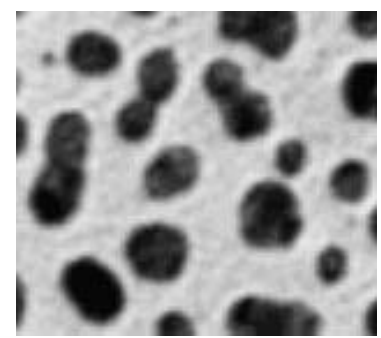

(a)

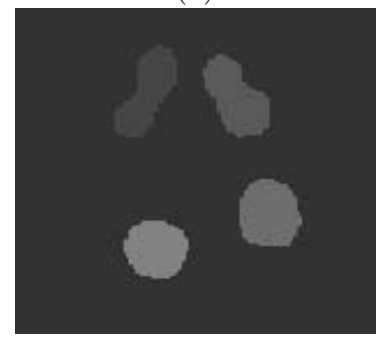

(c)

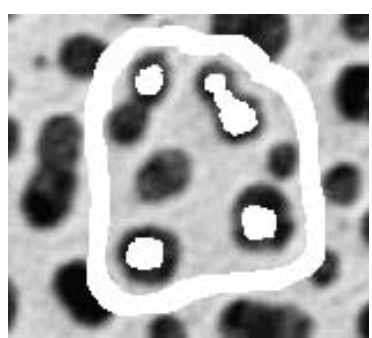

(b)

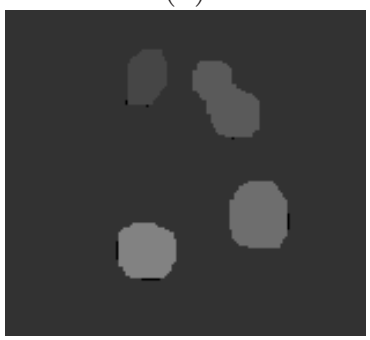

(d)

Fig. 5. (a) Original Image; (b) markers used (displayed in white) over the original image; (c) result of a watershed; and (d) result of modified flooding with $\widehat{\gamma}_{\lambda, g}$ (where $\lambda$ is equal to 5$)$.

computation of the influence zones of each connected component of the marker. As discussed, it is possible that some pixels do not belong to any influence zone because of the added criterion. Then, the gray-level case has been considered, and a modified flooding process arises that can be used to segment regions of interest with additional flexibility. We have shown a medical image example in which the modified flooding process permits to separate overlapping particles.

\section{References}

1. S. Beucher, F. Meyer: The morphological approach to segmentation: the watershed transformation, in book "Mathematical morphology in image processing" (Ed.: E. Dougherty), pp. 433-481, New York: Marcel Dekker, 1993.

2. J. Crespo, J. Serra, R. Schafer: Theoretical aspects of morphological filters by reconstruction. Signal Process., 47(2), 201-225, 1995.

3. J. Crespo, R. Schafer: Locality and adjacency stability constraints for morphological connected operators, in Journal of Mathematical Imaging and Vision, vol. 7, pp. 85-102, 1997.

4. J. Crespo, V. Maojo: New Results on the Theory of Morphological Filters by Reconstruction, in Journal "Pattern Recognition". Vol. 31, Nro. 4, pp. 419-429, April 1998.

5. H. Digabel, C. Lantuéjoul: C. Iterative algorithms. Second Symposium Européen d'Analyse Quantitative des Microstructures en Sciences des Matériaux, Biologie et Médecine, Caen. J.-L. Chermant, Ed., Riederer Verlag, Stuttgart, pp. 85-99, 1977. 
6. H. Heijmans: Morphological Image Operators (Advances in Electronics and Electron Physics, Series Editor: P. Hawkes), Academic Press, 1994.

7. F. Meyer, S. Beucher: Morphological segmentation. J. Visual Commun. Image Repres, Volume 1, Number 1, Pages 21-45, 1990.

8. P. Salembier, J. Serra: Flat zones filtering, connected operators, and filters by reconstruction, in J. of IEEE Transactions on Image Processing, Vol. 4, pp. 11531160, 1995.

9. M. Schmitt, J. Mattioli: Morphologie Mathematique, Masson, 1993.

10. J. Serra: Image Analysis and Mathematical Morphology, Vol. 1, Academic Press, 1982.

11. J. Serra (Ed.). Image Analysis and Mathematical Morphology, Vol. 2, Academic Press, 1988.

12. J. Serra, Ph. Salembier: Connected operators and pyramids. In SPIE, editor, Proc. Image Algebra Math. Morphology, Volume 2030, pages 85-76, San Diego (CA), USA, July, 1993.

13. J. Serra: Connectivity on complete lattices. Journal of Mathematical Imaging and Vision, Volume 9, Pages 231-251, 1998.

14. P. Soille: Morphological Image Analysis: Principles and Applications, Springer, 2nd edition, 2003.

15. I. R. Terol, D. Vargas: A study of openings and closings with reconstruction criteria. In H. Talbot, and R. Beare, Editors. Mathematical Morphology, Proc. of the VIth International Symposium, 2002.

16. L. Vincent, P. Soille: Watersheds in Digital Spaces: An Efficient Algorithm Based on Immersion Simulations, J. IEEE Trans. Pattern Anal. Machine Intell, Volume 13, Pages 583-598, June 1991.

17. D. Vargas, J. Crespo, V. Maojo, I.R. Terol: Medical Image Segmentation Using Openings and Closings with Reconstruction Criteria, to be published in Proceedings of the International Conference on Image Processing ICIP, September 2003. 